\title{
Saúde e nutrição de crianças de 0 a 60 meses de um assentamento de reforma agrária, Vale do Rio Doce, MG, Brasil
}

\author{
Health and nutrition of children, 0 to 60-month old, in an \\ agrarian-reform settlement, Vale do Rio Doce, MG, Brazil
}

\author{
Teresa Gontijo de CASTRO' \\ Flávia Milagres CAMPOS ${ }^{1}$ \\ Silvia Eloiza PRIORE ${ }^{1}$ \\ France Maria Gontijo COELHO 2 \\ Maria Teresa Fialho de Sousa CAMPOS ${ }^{1}$ \\ Sylvia do Carmo Castro FRANCESCHINI ${ }^{1}$ \\ Arquimedes de Almeida RANGEL ${ }^{3}$
}

\section{RE S U M O}

\section{Objetivo}

Este estudo avaliou as condições gerais de saúde das crianças de um assentamento rural, localizado no Município de Tumiritinga, Vale do Rio Doce, MG, Brasil.

\section{Métodos}

Foram obtidos dados de 69 crianças de zero a 60 meses de idade, com relação ao estado nutricional (antropometria e nível de hemoglobina), parasitose intestinal, tempo de aleitamento materno, práticas alimentares e calendário de vacinação.

\section{Resultados}

Verificou-se que 7,6\% das crianças apresentavam déficits nos índices peso/idade e estatura/idade e 47,5\%, baixa concentração de hemoglobina. A infestação parasitária foi detectada em 96,1\% das 51 crianças que fizeram o exame e 34,0\% estavam com a vacinação atrasada. A mediana de tempo de aleitamento materno exclusivo foi de 30 dias e observou-se inadequada freqüência de consumo de alimentos-fontes de ferro.

\footnotetext{
1 Departamento de Nutrição e Saúde, Universidade Federal de Viçosa. Campus Universitário, s/n, 36571-000, Viçosa, MG,

Brasil. Correspondência para/ Correspondence to: S.E. PRIORE. E-mail: sepriore@ufv.br

2 Departamento de Economia Rural, Universidade Federal de Viçosa, MG, Brasil.

3 Prefeitura Municipal de Tumiritinga, MG, Brasil.
} 


\section{Conclusão}

Dado o aumento dos assentamentos rurais no Brasil e as precárias condições de saúde e nutrição infantil encontradas neste estudo, tornam-se necessárias medidas urgentes e efetivas de intervenção preventiva, voltadas à promoção da saúde dessa população.

Termos de indexação: estado nutricional, anemia, antropometria, aleitamento materno, assentamentos rurais.

\section{A B S T R A C T}

\section{Objetive}

This study evaluated the general health conditions of children living in a rural settlement in Tumiritinga, Vale do Rio Doce, MG, Brazil.

\section{Methods}

The obtained data regards the nutritional state (anthropometry and hemoglobin level), intestinal parasitic infestation, breast-feeding period, food habits and vaccination records of 69 children, ranging from zero to 60 months of age.

\section{Results}

The results showed that $7.6 \%$ of the children presented low indices of weight/age and height/age correlations, and $47.5 \%$ presented low hemoglobin level. Parasitic infestation was detected in $96.1 \%$ of the 51 children examined, and $34,0 \%$ had incomplete vaccination records. The average period of exclusive breast-feeding was 30 days. The consumption of iron-rich foods was also found inadequate.

\section{Conclusion}

Given the increasing number of rural settlements in Brazil and given the poor health and nutritional circumstances of the children studied in this work, urgent and effective measures of preventive intervention must be taken to promote this population's health conditions.

Index terms: nutritional state, anemia, anthropometry, breast-feeding, rural settlements.

\section{N T R O D U Ç Ã O}

O Movimento dos Trabalhadores Sem Terra (MST) teve início em 1979, em decorrência do processo de expropriação do campo e das crises econômica e de empregos nos centros urbanos, agravados pela crise energética mundial da década de 70. Diante da situação e das condições a que ficaram expostos, os trabalhadores rurais, para se manterem no campo, buscaram formas de resistência, iniciando a luta pela terra'.

Em Minas Gerais, as atividades de reflexão do movimento começaram dentro das Comunidades Eclesiais de Base e, em 1993, foi ocupada a fazenda Califórnia, no município de Tumiritinga, dando origem ao Assentamento Primeiro de Junho, cujo nome alude à data da ocupação. Em 1997², foram assentadas 82 famílias no local, apesar de o INCRA haver indicado que deveriam ser 56 . Até a conquista definitiva das terras do Primeiro de Junho, as famílias que hoje o compõem vieram seguindo uma trajetória de luta, opressão, fome e miséria.

Quanto à forma de organização das famílias e da produção, depois de instalado o assentamento, ficou acertado que uma área da fazenda ficaria para o chamado grupo dos 
coletivos e outra para o dos individuais. Os coletivos uniram seus lotes, fazendo uso comum da terra e dos créditos obtidos, organizando-se por setores de produção e dividindo tarefas. Os individuais optaram por manter seus lotes separados entre si, trabalhando na forma de produção familiar².

A desnutrição infantil no Brasil diminuiu entre as décadas de 70 e 90, como mostram os levantamentos nacionais de 1975, 1989 e 1996; no entanto, nos dados obtidos em 1989 e 1996, observa-se que este declínio foi menos acentuado nas áreas rurais do que nas urbanas do país ${ }^{3}$. Quando a concentração de hemoglobina cai abaixo de dois desvios-padrão da média de concentração numa população normal, do mesmo sexo, idade e vivendo na mesma altitude, considera-se que a anemia está presente. Esta pode ser causada por hemólise excessiva, defeitos congênitos na síntese de hemoglobina, perda crônica de sangue, hemorragia, deficiência de ferro, vitamina $B_{12}$, ácido fólico, vitamina $A$ e vitamina $C$. No entanto, a deficiência de ferro é responsável pela maioria das anemias que ocorrem em locais onde prevalece esta carência ${ }^{4}$.

No grupo infantil, a principal causa de anemia é a alimentação inadequada, uma vez que agravam o quadro de anemia tanto o desmame precoce, quanto a introdução de alimentos inapropriados, associados à manutenção de dietas basicamente lácteas, sem o suficiente consumo de alimentos fontes de ferro e com alta biodisponibilidade 5 . O Ministério da Saúde preconiza, como atenção básica de saúde para crianças, o acompanhamento do crescimento e do desenvolvimento, o incentivo ao aleitamento materno e o controle das imunizações e da infestação parasitária, das diarréias e das infecções respiratórias agudas (IRA) ${ }^{3}$.

Diante das situações nutricionais encontradas nacionalmente e do pouco que se conhece sobre a saúde e nutrição de crianças que residem em assentamentos rurais, este estudo objetivou avaliar o estado nutricional e a concentração de hemoglobina de crianças de zero a 60 meses do Assentamento Primeiro de Junho, além de avaliar alguns fatores intercorrentes à saúde deste grupo etário.

\section{CASUÍSTICA E MÉTODOS}

Trata-se de um estudo descritivo de delineamento transversal, no qual foram estudadas $100 \%$ das crianças de zero a 60 meses $(n=69)$, residentes no Assentamento Primeiro de Junho, localizado no município de Tumiritinga, na região do Vale do Rio Doce, em Minas Gerais, Brasil. Estas crianças pertenciam a 43 famílias do assentamento $(54 \%$ do total de famílias residentes), incluindo tanto as do módulo de organização individual, como as do coletivo.

Para verificar a existência de anemia, a avaliação da concentração de hemoglobina foi feita com hemoglobinômetro portátil por meio de punção digital do dedo anular da mão nãodominante ou de punctura do pé nas crianças que ainda não andavam. O ponto de corte adotado para anemia foi para valores menores que $11 \mathrm{~g} / \mathrm{dL}$, segundo o estabelecido pela Organização Mundial de Saúde ${ }^{6}$. As crianças anêmicas receberam tratamento medicamentoso prescrito pelo pediatra local e fornecido pela Prefeitura Municipal de Tumiritinga, MG.

Para avaliar o estado nutricional das crianças com dois anos ou mais, estas foram pesadas utilizando-se balança portátil digital eletrônica (com capacidade de $150 \mathrm{~kg}$ e sensibilidade de $50 \mathrm{~g}$ ) e foram medidas em sua altura com fita métrica metálica (com extensão de 2 metros, dividida em centímetros e subdividida em milímetros). $\mathrm{Na}$ avaliação das crianças menores de dois anos, utilizou-se a balança pesa-bebê (com capacidade de $16 \mathrm{~kg}$ e precisão de $10 \mathrm{~g}$ ) e mediu-se o comprimento com um antropômetro infantil. As técnicas utilizadas na obtenção do peso e estatura foram às propostas por Jelliffe ${ }^{7}$. A determinação dos índices peso/idade (P/I), peso/estatura (P/E) e estatura/idade (E/I) foi feita pelo critério escore-Z, considerando-se a referência do National Center for Health Statistics (NCHS) ${ }^{8}$. 
A desnutrição foi indicada pelo escore-Z s-2 para os índices peso/idade, estatura/idade ou peso/estatura; o risco nutricional para desnutrição foi indicado pelo escore-Z $>-2$ e $\leq-1$. O indicador de sobrepeso foi o escore- $Z \geq+2$ para peso/idade ou peso/estatura; o indicador de risco nutricional para sobrepeso foi o intervalo $\geq+1$ e $<+2$.

Exames parasitológicos de fezes (pesquisa de helmintos e protozoários) foram realizados em laboratório de análises clínicas indicado pela Prefeitura Municipal de Tumiritinga, MG. Foram submetidas ao exame 51 crianças, e as parasitadas receberam medicamento apropriado fornecido pela Prefeitura e prescrito pelo pediatra local.

O exame parasitológico não foi realizado em 18 crianças, porque sete delas tinham menos de 6 meses de idade e onze tiveram dificuldade de coleta do material fecal na data prevista para o estudo.

Por meio de inquérito, obtiveram-se informações referentes às características sociais das famílias (escolaridade dos pais, número de filhos, número de cômodos e fornecimento de água tratada no domicílio, forma de organização e produção), informações biológicas sobre as crianças de zero a 60 meses (peso ao nascer, tipo de parto, idade gestacional), e sobre as práticas alimentares, aleitamento materno e introdução de alimentos. Às mães com crianças maiores de 1 ano aplicou-se também questionário de freqüência de consumo alimentar, enquanto no inquérito destinado às mães de menores de 1 ano, acrescentaram-se algumas questões sobre a época de introdução de alimentos.

Verificou-se a vacinação diretamente no Cartão da Criança; as mães daquelas que estavam com a vacinação atrasada, foram orientadas a procurar o Centro de Saúde do município para atualizá-la. Utilizou-se como referência o calendário básico de imunizações recomendado pelo Ministério da Saúde ${ }^{9}$, excetuando-se as vacinas contra hepatite B e Haemophillus influenzae, que foram desconsideradas pelo fato de terem sido incluídas há apenas alguns anos e estarem disponíveis, exclusivamente, para menores de 2 anos de idade.

Os dados obtidos foram avaliados com o auxílio do software Epi Info, versão 6.01, e as associações foram feitas pelo teste do quiquadrado, a partir de tabelas $2 \times 2$, sendo considerados estatisticamente significantes os valores menores ou iguais a $5 \%$ ou $0,05(p \leq 0,05)$.

\section{RESULTADOS}

Com relação à forma de ocupação e organização, 44\% das famílias eram individuais e $56 \%$, coletivas. Um dos núcleos de habitação do assentamento, a Agrovila, não possuía rede de esgoto, razão pelo qual todos os moradores, coletivos e parte dos individuais, faziam uso de fossas. O assentamento denominado Limeira, exclusivo dos moradores individuais, não possuía fossa e o esgoto era lançado em córregos próximos.

Tabela 1. Características sociais das famílias das crianças de zero a 60 meses do Assentamento Primeiro de Junho. Tumiritinga, MG, Brasil, 2001.

\begin{tabular}{lcc}
\hline \multirow{2}{*}{ Características } & \multicolumn{2}{c}{ Freqüência } \\
\cline { 2 - 3 } & \multicolumn{1}{c}{$\mathrm{n}$} & $\%$ \\
\hline Fornecimento de água & 34 & 79,0 \\
Poços/minas & 9 & 21,0 \\
Escolaridade formal do pai* & & \\
$\leq 4$ anos & 35 & 85,4 \\
$>4$ anos & 6 & 14,6 \\
Escolaridade formal da mãe & & \\
$\leq 4$ anos & 31 & 72,0 \\
$>4$ anos & 12 & 28,0 \\
Número de filhos & & \\
1 & 8 & 18,6 \\
2 & 12 & 27,9 \\
3 & 6 & 14,0 \\
4 ou mais & 17 & 39,5 \\
Número de quartos por domicílio & & \\
$\leq 2$ & 22 & 51,2 \\
$>2$ & 21 & 48,8 \\
\hline & &
\end{tabular}

${ }^{(*)}$ Duas mães não souberam informar a escolaridade do pai da criança. 
Apesar de 79\% dos domicílios terem abastecimento com água tratada, o uso de água proveniente de poços e minas era comum entre os assentados, principalmente entre os individuais do Limeira (Tabela 1).

O baixo nível de instrução foi mais pronunciado no sexo masculino, sendo a escolaridade de $85,4 \%$ dos pais menor ou igual a 4 anos de ensino formal, e $72,0 \%$ das mães.

Observou-se, ainda, a existência de famílias numerosas, considerando que 39,5\% destas tinham 4 filhos ou mais, sendo a média de 3,7 filhos/mulher (mínimo de 1, máximo de 14 e mediana de 3 filhos). Aliado ao maior número de filhos, verificou-se que cerca de 50,0\% dos domicílios possuía em média dois quartos, o que contribuía para a aglomeração de pessoas em um mesmo cômodo.

Das crianças de zero a 60 meses, $62,0 \%$ pertenciam ao sexo masculino. A distribuição etária era de $17,0 \%$ menores ou iguais a 1 ano e o restante $(83,0 \%)$, acima desta idade. Verificou-se que $88,2 \%$ nasceram de parto normal, $97,0 \%$ a termo e 93,0\% com peso normal, ou seja, apresentaram boas condições ao nascimento.

No mês anterior ao estudo, 62,0\% das crianças, segundo as mães, apresentaram alguma enfermidade, e o tratamento mais empregado foi o consumo de chás (hortelã, poejo, erva-cidreira), mencionado por $69,8 \%$, enquanto as práticas medicamentosas convencionais foram adotadas em $30,2 \%$ dos casos. Das doenças referidas pela população, a maior parte estava relacionada com o aparelho respiratório, diarréia e desidratação apresentadas no último mês somaram, juntas, $18,0 \%$ das enfermidades que mais acometeram o grupo no respectivo período.

Cerca de 34\% das crianças não estavam com a vacinação em dia. As doses em atraso foram referentes à febre amarela (35\%), tríplice-DPT (29\%), antipóliomielite (26\%) e sarampo (10\%). Este percentual de atraso na vacinação poderia ser ainda maior, caso as vacinas contra hepatite $B$ e Haemophillus influenzae fossem consideradas.

Em relação à prevalência de anemia, a freqüência de crianças de 6 a 60 meses com baixa concentração de hemoglobina foi de $47,5 \%$. 0 valor médio de hemoglobina para as menores de 6 meses foi de 10,7g/dL ( $n=3)$ e, para as maiores, de $10,86 \mathrm{~g} / \mathrm{dL}(\mathrm{n}=61)$. Distribuiram-se as crianças anêmicas maiores de seis meses, de acordo com a faixa etária. Observou-se a diminuição do percentual de anêmicos à medida que aumentou a idade das crianças (Tabela 2).

Foi encontrada associação entre a anemia e o atraso no calendário de vacinações ( $p=0,0041)$.

A distribuição das crianças de zero a 60 meses, quanto aos intervalos de escore-Z, indicou prevalência, acima do esperado na distribuição normal, para desnutrição (índices P/I e E/l) e para o risco nutricional para desnutrição (P/E, P/l e E/l) (Tabela 3).

A maior taxa de desnutrição foi na faixa etária de 1 a 2 anos, 22,2\% e 33,0\% para P/l e $\mathrm{E} / \mathrm{l}$, respectivamente. A associação entre o baixo

Tabela 2. Crianças com baixa concentração de hemoglobina $(<11 \mathrm{~g} / \mathrm{dL})$, segundo a faixa etária, Assentamento Primeiro de Junho, Tumiritinga, MG, Brasil, 2001.

\begin{tabular}{lccc}
\hline Faixa etária (meses) & Total* $^{*}(\mathrm{n})$ & Anêmicos $(\mathrm{n})$ & Percentual de anêmicos (\%) \\
\hline $6-23$ & 14 & 10 & 71,4 \\
$24-35$ & 16 & 10 & 62,5 \\
$36-60$ & 31 & 9 & 29,0 \\
\hline Total & 61 & 29 & 47,5 \\
\hline
\end{tabular}

${ }^{(*)}$ As crianças menores de 6 meses foram excluídas desta análise pelo fato de não existir ponto de corte para anemia nesta idade; 1 criança maior de 6 meses não fez a medida de hemoglobina. 
peso ao nascer $(<2500 \mathrm{~g})$ e a desnutrição (índice $\mathrm{P} / \mathrm{l})$ foi verificada $(p=0,0006)$.

Associações do baixo nível de escolaridade materna, tanto com a desnutrição como com a anemia, não foram encontradas.

Constatou-se a prevalência de parasitose em cerca de $96,1 \%$ das 51 crianças de zero a 5 anos, que fizeram o exame parasitológico; boa parte delas estava poliparasitada (em 56,6\% foram detectados dois ou mais parasitas). Os parasitas mais encontrados foram Ascaris lumbricoides (77,6\%), Entamoeba histolytica (42,9\%) e Giardia lamblia (34,7\%).

Apesar da prevalência de parasitose, sua associação com a anemia só foi verificada em relação à Entamoeba histolytica $(p=0,0319)$.

Tabela 3. Distribuição percentual das crianças de zero-60 meses quanto aos intervalos de faixa etária e de escore-Z, segundo os índices P/I, P/E e E/I. Assentamento Primeiro de Junho, Tumiritinga, MG, Brasil, 2001.

\begin{tabular}{|c|c|c|c|c|c|c|c|c|c|c|c|c|}
\hline \multirow{2}{*}{ Índice } & \multirow{2}{*}{$\begin{array}{c}\text { Idade } \\
\text { (meses) }\end{array}$} & \multirow[b]{2}{*}{$\mathrm{n}$} & \multicolumn{2}{|c|}{$\leq-2 z$} & \multicolumn{2}{|c|}{$>-2 z$ e $\leq-1 z$} & \multicolumn{2}{|c|}{$>-1 z e<+1 z$} & \multicolumn{2}{|c|}{$\geq+1 z e<+2 z$} & \multicolumn{2}{|c|}{$\geq+2 z$} \\
\hline & & & $\%$ & $n$ & $\%$ & $n$ & $\%$ & $n$ & $\%$ & $\mathrm{n}$ & $\%$ & $n$ \\
\hline \multirow[t]{5}{*}{$\mathrm{P} / \mathrm{I}$} & $0-11$ & 10 & - & & - & & 70,0 & $(7)^{a}$ & 30,0 & (3) & - & \\
\hline & $12-23$ & 9 & 22,2 & (2) & 33,3 & (3) & 44,5 & (4) & - & & - & \\
\hline & $24-35$ & 16 & 6,3 & (1) & 31,1 & (5) & 50,0 & (8) & 6,3 & (1) & 6,3 & (1) \\
\hline & $36-47$ & 22 & 9,1 & $(2)$ & 50,0 & (11) & 27,3 & (6) & 13,6 & (3) & - & \\
\hline & $48-60$ & 9 & - & & 33,3 & (3) & 44,5 & (4) & 22,2 & (2) & - & \\
\hline \multirow[t]{5}{*}{ P/E } & $0-11$ & 10 & - & & 0,0 & (0) & 100,0 & (10) & - & & - & \\
\hline & $12-23$ & 9 & - & & 22,2 & (2) & 77,8 & (7) & - & & - & \\
\hline & $24-35$ & 16 & - & & 31,1 & (5) & 68,9 & (11) & - & & - & \\
\hline & $36-47$ & 22 & - & & 22,7 & (5) & 50,0 & (11) & 27,3 & (6) & - & \\
\hline & $48-60$ & 9 & - & & - & - & 100,0 & (9) & - & & - & \\
\hline \multirow[t]{5}{*}{$E / l^{b}$} & $0-11$ & 10 & - & & 10,0 & (1) & 60,0 & (6) & 30,0 & (3) & - & \\
\hline & $12-23$ & 9 & 33,3 & (3) & 11,1 & (1) & 55,6 & (5) & - & & - & \\
\hline & $24-35$ & 16 & - & & 31,1 & (5) & 43,8 & (7) & 18,8 & (3) & 6,3 & (1) \\
\hline & $36-47$ & 22 & 9,1 & (2) & 36,4 & (8) & 45,5 & (10) & 4,5 & (1) & 4,5 & (1) \\
\hline & $48-60$ & 9 & - & & 33,3 & (3) & 66,4 & (6) & - & & - & \\
\hline
\end{tabular}

$\mathrm{n}=66$

(a) Números entre parênteses representam o " $\mathrm{n}$ " de cada faixa de escore- $Z$.

(b.) $\mathrm{O}$ índice estatura/idade (E/I) foi estratificado nas faixas de desvio-padrão $\geq+1 z$ e $<+2 z$; $\geq+2 z$ para facilitar a compreensão da tabela, no entanto, ressalta-se que os valores >-1z são considerados eutrofia.

Tabela 4. Freqüência de consumo de alimentos pelas crianças maiores de 1 ano de idade, Assentamento Primeiro de Junho, Tumiritinga, MG, Brasil, 2001

\begin{tabular}{lcccc}
\hline & \multicolumn{4}{c}{ Freqüência de Consumo (\%) } \\
\cline { 2 - 5 } Tipo de Alimento & 1 a 3 vezes / semana & 4 a 7 vezes / semana & $<1$ vez / semana & Não come \\
\hline Cereais & 7,3 & 92,7 & - & - \\
Leguminosas & 5,5 & 94,5 & - & - \\
Leite & 13 & 79,6 & 7,4 & 16,3 \\
Hortaliças & 23,6 & 52,7 & 25,5 & - \\
Frutas & 38,1 & 36,4 & 18,2 & 3,6 \\
Carnes & 50,9 & 27,3 & & \\
\hline
\end{tabular}

$\mathrm{n}=54$ 
Em relação às práticas de aleitamento materno, hábito e consumo alimentar, foi relatado o uso de chás desde os primeiros dias de vida da criança. Nas maiores de 1 ano, o consumo dessas infusões também foi mencionado em 96,4\%; destas, 51,0\% o faziam apenas em situações em que apresentavam alguma enfermidade, enquanto 49,0\% tinham o hábito de ingeri-lo freqüentemente. Não se encontrou associação entre o consumo de chás e a anemia, apesar da possibilidade de fatores antinutricionais interferirem na biodisponibilidade de ferro.

Os alimentos consumidos pelas crianças maiores de 1 ano, com maior freqüência eram as leguminosas, particularmente o feijão e o arroz, os cereais e o leite. As hortaliças, frutas e carnes apresentaram baixo consumo ou estavam ausentes na alimentação diária (Tabela 4).

Ao avaliar o consumo de guloseimas (balas, chicletes, pipocas, salgadinhos e pirulitos), verificou-se que este hábito era freqüente $(67,3 \%$ o faziam pelo menos 1 vez por semana).

Com relação ao aleitamento materno total, a mediana de tempo foi de 12 meses; no entanto, o aleitamento realizado de forma exclusiva, foi de 30 dias. Foi relatada a introdução de alimentos antes dos 6 meses de vida para todas as crianças menores de 1 ano.

O tempo de aleitamento materno exclusivo não mostrou associações nem com a anemia, nem com a desnutrição.

\section{I S C U S S Ã O}

As condições de saneamento encontradas no assentamento não foram favoráveis, visto que não havia rede de esgotos; além disso, o percentual de domicílios providos de água tratada era bem inferior ao percentual de $90 \%$ de fornecimento de água tratada em centros urbanos $^{10}$, sendo comum, no assentamento, o uso de minas e poços. Como agravante, o fato de não haver rede de esgotos aumentava o risco de contaminação da água utilizada nos domicílios ${ }^{10}$.
A escolaridade paterna e materna foi inferior à média nacional, que é de 5,9 e 6,8 anos, respectivamente ${ }^{10}$. A maior escolaridade está diretamente relacionada com a maior compreensão da etiologia das doenças infantis, a maior eficiência dos cuidados higiênicos com a criança e a melhor utilização dos serviços públicos de saúde ${ }^{11}$.

O número médio de filhos por família, superou o valor estimado para a população brasileira, que é de 2,5 filhos por mulher ${ }^{12}$.

Aliadas às doenças respiratórias, a diarréia e a desidratação foram as enfermidades mais freqüentemente relatadas pelas mães das crianças ( $9 \%$, cada uma). O elevado percentual de crianças com doenças respiratórias, detectado no assentamento, comprova a afirmação da Organização Pan-Americana de Saúde, ${ }^{13}$ segundo a qual, as infecções respiratórias agudas constituem um dos principais problemas de saúde que afetam crianças menores de 5 anos na América Latina. Tais infecções são causa, não só de um número significativo de consultas, como também de hospitalização, seqüelas e mortalidade, além de contribuir para o uso inapropriado de antibióticos e outros medicamentos. A diarréia também resulta em considerável morbimortalidade em menores de 5 anos de idade. Sabe-se que nos países do terceiro mundo, a diarréia é a causa mais provável da morte de crianças até dois anos de idade, já que a desidratação, dada como a principal causa de internação e morte, é conseqüência da diarréia aguda ${ }^{14}$.

Os valores de cobertura vacinal encontrados revelaram-se abaixo da média divulgada pelo Ministério da Saúde, para todo o território nacional, que era de $98 \%$ contra sarampo, 94\% contra difteria, coqueluche e tétano (tríplice-DPT) e $98 \%$ contra poliomielite, no ano de 1999, em crianças menores de um ano ${ }^{9}$. O considerável atraso no calendário de vacinação das crianças pode, em parte, ser justificado pela forma como o posto de saúde do município, onde se localiza o assentamento, administra a aplicação das vacinas. Pelo fato de o município ter uma população pequena 
(menos de 6500 habitantes), a administração do posto deixa que se acumule certo número de crianças e então marca um dia para os pais levarem seus filhos, que muitas vezes, diante de imprevisto, não comparecem. Além disto, para levar as crianças ao posto de saúde, muitas vezes os pais têm de perder todo um dia de trabalho, ou parte dele, devido à distância entre o assentamento e o município.

A prevalência de anemia no grupo de 6 a 60 meses foi de 46,9\%, semelhante à encontrada por Monteiro et al. ${ }^{15}$ em estudo realizado em 1996, no município de São Paulo. Como verificado no referido estudo, o risco desta desordem foi alto no primeiro ano de vida, mantendo-se elevado no segundo e retrocedendo, gradualmente, a partir do terceiro.

Com relação ao estado nutricional, a prevalência de desnutrição foi menor que a encontrada na avaliação do estado nutricional de crianças de zero a 10 anos, residentes em ocupação dos "sem-terra" em Porto Calvo, Alagoas. Ali, as prevalências de déficits nutricionais, relativos ao peso para idade, estatura para idade e peso para estatura, foram, respectivamente, de $19,6 \%, 39,8 \%$ e $2,0 \%{ }^{16}$. Já em um estudo realizado com residentes de um assentamento rural no Rio de Janeiro, não foram encontrados déficits nutricionais, relativos aos índices acima citados, em nenhuma das crianças com idade inferior a 5 anos. Contudo, ressalta-se que o referido estudo foi realizado em um assentamento consolidado há mais tempo, cuja proximidade com centros urbanos facilita o acesso aos serviços de saúde. Além disso, a porcentagem de crianças vacinadas e o tempo de aleitamento materno exclusivo foram superiores aos encontrados no presente estudo ${ }^{17}$.

Na população estudada, o percentual de desnutridos para o índice E/I encontrado, foi de 7,6\%; menor que média nacional, de 10,5\%, para crianças menores de 5 anos $^{18}$. O maior percentual de desnutrição foi encontrado na faixa de 1 a 2 anos; tal tendência já fora também observada pela Pesquisa Nacional sobre Demografia e Saúde
$(P N D S)^{18}$, segundo a qual, a partir dessa faixa etária a criança parece melhorar.

Constatou-se neste estudo, a associação entre baixo peso ao nascer $(<2500 \mathrm{~g})$ e desnutrição. Assis \& Barreto $^{14}$ também relataram ter encontrado uma incidência mais elevada de desnutrição, segundo o índice peso/idade, em crianças até 5 anos, nascidas pré-termo, com baixo peso $(<2500 \mathrm{~g})$ e com menos de $46 \mathrm{~cm}$ de comprimento.

A associação da anemia com a infestação pela Entamoeba hystolitica, provavelmente deve-se ao fato de este parasita geralmente desencadear quadros de diarréia e má absorção de ferro, conforme relatado por Ferreira et al. ${ }^{19}$, o que estaria, conseqüentemente, reduzindo os estoques orgânicos de ferro. A não-associação dos demais parasitas com a anemia, talvez decorra de não estarem tais parasitas envolvidos na espoliação sangüínea, comumente causada pelo Ancylostoma duodenale e pelo Necator americanus ${ }^{20}$.

Como a carência de ferro é a principal causa de anemia, e a carne é o único alimento fonte do ferro hemínico, o baixo consumo de carnes pela população pode ter contribuído para a grande incidência de anemia. No entanto, não foi evidenciada associação significante entre o baixo consumo de carnes e a anemia. Aliado a este fato, o consumo insuficiente de frutas e hortaliças pode agravar o estado nutricional também quanto ao ferro. Sabe-se que nestes grupos de alimentos se encontram as fontes de vitamina $C$ (frutas) e de vitamina $A$ (frutas e hortaliças), vitaminas importantes no processo de biodisponibilidade do ferro dietético não-hemínico, aumentando sua absorção. É importante ressaltar que o assentamento em questão localiza-se em uma área altamente degradada e com insolação intensa. Tais limitações ambientais podem ser a causa do baixo consumo de frutas e hortaliças pela população, já que a produção agrícola é a base da alimentação das famílias.

O consumo de guloseimas chamou a atenção, pois, mesmo estando em área rural, as 
crianças tinham freqüentemente acesso a esse tipo de alimento. Apesar da avaliação da ingestão ter sido qualitativa e não quantitativa, e de não ser possível analisar o quanto o consumo das guloseimas estaria interferindo na saúde das crianças, observou-se, contudo, haver um erro alimentar, visto que parte do orçamento familiar é destinado à compra de guloseimas, enquanto se negligencia a aquisição de frutas e hortaliças.

Para as crianças menores de um ano, o pouco tempo de aleitamento materno exclusivo, aliado ao maior risco de desenvolvimento de anemia nesta faixa etária, talvez explique a prevalência de anemia (100\%), embora, neste estudo, não se tenha encontrado associação entre anemia e tempo de aleitamento materno exclusivo.

O aleitamento materno exclusivo nos primeiros seis meses de vida é tido como um fator protetor contra a anemia, pois, embora o leite materno contenha quantidades pequenas de ferro, a sua alta biodisponibilidade garante que as necessidades de ferro até os seis meses de vida sejam supridas 4 .

Segundo Silva et al..$^{21}$, crianças menores de dois anos são mais vulneráveis à anemia, devido à prevalência do desmame precoce no País, ao atraso na introdução de alimentos ricos em ferro na dieta da criança, à maior velocidade de crescimento nessa faixa etária e à maior prevalência de doenças como diarréia e infecções respiratórias nos primeiros anos de vida.

\section{RE C O MEN D A ÇÕ ES}

O número crescente de assentamentos rurais no Brasil suscita a necessidade de mais estudos sobre as condições de saúde da população infantil residente nesses locais, para possibilitar o diagnóstico e a análise da realidade, de forma a propor intervenções mais eficazes. Além disso, o histórico e a caracterização socioeconômica e ambiental dos assentamentos, tornam-se igualmente importantes nesses estudos.
No que se refere à população menor de 5 anos, a avaliação do estado nutricional, análise das práticas de alimentação no primeiro ano de vida, exames parasitológicos de fezes, observação do calendário de vacinação e medidas profiláticas contra a anemia devem ser priorizadas no trabalho em assentamentos rurais.

\section{A GRADECIMENTOS}

Os autores agradecem à comunidade do Assentamento Primeiro de Junho pela receptividade e pela colaboração com o trabalho realizado.

\section{RE FER Ê NCIAS}

1. Souza MJ. Uma Educação do Campo: o ensino fundamental em um assentamento na região do Rio Doce-MG [monografia]. Viçosa: Departamento de Educação, Universidade Federal de Viçosa; 2000. $37 p$.

2. Coelho FMG, Botelho MIV. Individual, coletivos e semi-coletivos: autonomia individual e referência coletiva. In: Anais do $10^{\circ}$ Congresso Internacional de Sociologia Rural; 2000. Rio de Janeiro: Sociedade Brasileira de Economia Rural; 2000. p.54-66.

3. Brasil. Ministério da Saúde. Atenção Integrada às Doenças Prevalentes na Infância [online] [citado 2001 mai 15]. Disponível em: http://www.saude. gov.br/programas/scriança/criança/aidpi/aidpi1. htm

4. World Health Organization. Iron deficiency anaemia: assessment, prevention and control. A guide for programme managers. Geneva; 2001. 114p. Document WHO/NHD/01.3.

5. Schimitz BAS, Picanço MR, Aquino KKNC, Bastos J, Giorgini E, Cardoso R, et al. A prevalência de desnutrição e anemia em pré-escolares de Brasília - Brasil. Pediatr Mod 1998; 34(4):155-64.

6. Organização Mundial de Saúde. Anemias nutricionais. Genebra; 1972. 32p. (Série de Informes Técnicos, 405). 
7. Jelliffe DB. Evaluación del estado de nutrición de la comunidad. Genebra: OMS; 1968. (Série de Monografias).

8. Organización Mundial de la Salud. Medición del cambio del estado nutricional. Ginebra; 1983.

9. Brasil. Ministério da Saúde. Fundação Nacional de Saúde. Programa Nacional de Imunização [online]. [citado 2001 ago 03]. Disponível em: http:// www.funasa.gov.br/ imu/imu01.htm

10. Instituto Brasileiro de Geografia e Estatística. Síntese de indicadores sociais, 2000. Rio de Janeiro; 2001.

11. Ribas DLB, Philippi ST, Tanaka ACDÁ, Zorzatto JR. Saúde e estado nutricional infantil de uma população da região Centro-oeste do Brasil. Rev Saúde Pública 1999; 33(4):358-65.

12. Patarra NL. Mudanças na dinâmica demográfica. In: Monteiro CA, organizador. Velhos e novos males da saúde no Brasil: a evolução do País e de suas doenças. 2.ed. São Paulo: Hucitec; 2000. p. 61-78.

13. Organização Pan-Americana de Saúde. Ações de Saúde Materno-Infantil a nível local: segundo as metas da Cúpula Mundial em favor da infância. Washington, DC; 1997. 283p.

14. Assis AM, Barreto ML. Condições de vida, saúde e nutrição na infância em Salvador. Brasília: INAN; 2000.165p.

15. Monteiro CA, Szarfarc SC, Mondini L. Tendência secular da anemia na infância na cidade de São
Paulo (1984-1996). Rev Saúde Pública 2000; 34(6):62-72.

16. Ferreira HS, Albuquerque MFM, Ataide TR, Morais MGC, Mendes MCR, Siqueira TCA, et al. Estado nutricional de crianças menores de 10 anos residentes em invasão do "Movimento dos Sem-Terra", Porto Calvo, Alagoas. Cad Saúde Pública 1997; 13(1):234-41.

17. Veiga GV, Burlandy L. Indicadores sócio-econômicos, demográficos e estado nutricional de crianças e adolescentes residentes em um assentamento rural do Rio de Janeiro. Cad Saúde Pública 2001; 17(6):1465-72.

18. Sociedade Civil do Bem-Estar no Brasil. Pesquisa Nacional sobre Demografia e Saúde (PNDS), 1996. Rio de Janeiro: Macro International; 1996.

19. Ferreira UM, Ferreira CS, Monteiro CA. Tendência secular das parasitoses intestinais na infância na cidade de São Paulo (1984-1996). Rev Saúde Pública 2000; 34:(6):73-82.

20. Vannucchi H, Freitas M, Szarfarc SC. Prevalência de anemias nutricionais no Brasil. Cad Nut 1992; 4:7-26.

21. Silva LSM, Giugliani ERJ, Aerts DRGC. Prevalência e determinantes de anemia em crianças de Porto Alegre, RS, Brasil. Rev Saúde Pública 2001; 35(1):66-73.

Recebido para publicação em 31 de janeiro de 2002 e aceito em 27 de fevereiro de 2003. 